\title{
Claves para comprender la resistencia de los colectivos antivacunas: una controversia científico-tecnológica pública*
}

Keys to understanding the resistance of anti-vaccination groups: a public scientific-technological controversy

\section{Obdulia Torres González ${ }^{* *}$}

\section{Resumen}

En este artículo se aborda el debate acerca de las vacunas como un caso de controversia científico tecnológica pública. En la controversia de las vacunas hay una cuestión científica, la efectividad de las vacunas en la eliminación de las enfermedades; una cuestión de evaluación de riesgos, los posibles efectos adversos y la posibilidad de que la inmunización cause enfermedades idiopáticas; una cuestión ética, el equilibrio de derechos entre los dos grupos y los límites de la libertad de elección de tratamiento; y una cuestión política, quien debe tomar las decisiones acerca de la inmunización y si esta debe ser obligatoria. El análisiss de la controversia da como resultado que es la cosmovisión del mundo, que proviene en gran parte de creencias New Age, sostenida por los grupos antivacunas, la que explica las actitudes de estos grupos hacia la vacunación. Esta cosmovisión provoca diferencias en la interpretación de la evidencia, de la ley, del riesgo y de la ciencia.

Palabras clave: antivacunas; riesgo; cosmovisión; expertos; Naturaleza.

\footnotetext{
* Recibido: julio 2018. Aceptado: julio 2018.

Este artículo se ha realizado en el marco del Proyecto de I+D "La frontera entre ciencia y política y la ciencia en la frontera: la ciencia española, 1907-1975”, FFI2015-64529-P (MINECO/FEDER). Agradezco a Ana Cuevas las sugerencias y comentarios a una versión preliminar de este artículo.

** Universidad de Salamanca,. Salamanca, España. Email: omtorres@usal.es
} 


\begin{abstract}
This article discusses the debate about vaccination as a case of public scientific technological controversy. In the vaccination controversy there is a scientific question, the effectiveness of vaccines in the elimination of diseases; a question of risk assessment, possible adverse effects and the possibility that immunization causes idiopathic diseases; an ethical question, the balance of rights between the two groups and the limits of the freedom of choice of treatment; and a political issue, who must take decisions about immunization and whether immunization should be mandatory. The results of the analysis of the controversy suggest that the attitudes of the anti-vaccination groups towards vaccination are largely explained by their worldview which comes majorly from New Age beliefs. This worldview causes differences in the interpretation of evidence, law, risk and science.
\end{abstract}

Keywords: Antivaccination; Risk; Worldview; Experts, Nature.

\title{
Introducción
}

La controversia de las vacunas lleva vigente desde el siglo XIX con las primeras reacciones frente a la vacunación obligatoria contra la viruela en Reino Unido. En este artículo se aborda este debate desde el marco teórico de las controversias científico tecnológicas públicas (CCTP). Este marco teórico nos permite dar cuenta de la controversia científica como tal, pero también del cúmulo de factores políticos, ideológicos, morales y valorativos que rodean este fenómeno. En la controversia de las vacunas hay una cuestión científica, la efectividad de las vacunas en la eliminación de las enfermedades; una cuestión de evaluación de riesgos, los posibles efectos adversos y la posibilidad de que la inmunización cause enfermedades idiopáticas; una cuestión ética, el equilibrio de derechos entre los dos grupos y los límites de la libertad de elección de tratamiento; y una cuestión política, quien debe tomar las decisiones acerca de la inmunización y si esta debe ser obligatoria.

El enfoque utilizado en este trabajo es una mezcla de las perspectivas acerca de las controversias (positivista, constructivista y de grupos políticos) analizadas por Martin y Richards (1995). Existe un amplio consenso científico respecto a la efectividad y seguridad de las vacunas y se intentará averiguar cuáles son las razones que llevan a los grupos de antivacunas a rechazar la inmunización (enfoque positivista). Pero se pretende analizar esta situación no desde una sociología del error, sino con un análisis equilibrado de ambos 
grupos, intentando determinar qué parte del conocimiento acerca del tema puede ser susceptible de más de una interpretación (enfoque de los grupos políticos). Se pretende asimismo mantener una cierta simetría a la hora de analizar ambos lados de la controversia (enfoque constructivista). El objetivo, por tanto, es analizar las posibles causas que hacen que la controversia de las vacunas no se cierre y reaparezca periódicamente con cada nueva noticia en los medios de comunicación relacionada con la cuestión.

La controversia es poliédrica, presenta muchas caras y muchas aristas. En primer lugar, existen distintos grupos con distintas actitudes hacia las vacunas. Nos vamos a centrar en aquel conjunto de individuos más recalcitrantes que no vacunan en ningún caso debido, fundamentalmente, a un conjunto de creencias o cosmovisiones acerca del mundo, el cuerpo humano y la naturaleza. Pero, incluso dentro de este reducido grupo, los actores se multiplican. En un lado de la controversia tenemos a la administración de salud pública con su pléyade de expertos agrupados en distintas asociaciones científicas y profesionales. Por otro lado, están los grupos antivacunas, que también conforman asociaciones, pero que hacen hincapié en distintos aspectos de la controversia. Podríamos decir que todos los actores opuestos a la vacunación comparten un conjunto de argumentos, aunque estos toman más o menos peso dependiendo de los objetivos en cada momento. Estos argumentos serían ${ }^{1}: 1$ ) las vacunas causan enfermedades idiopáticas como el autismo, la muerte súbita del lactante, disfunciones inmunológicas, desórdenes neurológicos, daño cerebral o déficit de atención, 2) la existencia de lotes de vacunas contaminados y la inmunización simultánea contra varias enfermedades aumenta el riesgo de padecer un efecto adverso, 3) las vacunas disminuyen la inmunidad, mientras las enfermedades que suceden naturalmente ayudan al sistema inmunológico a prevenir enfermedades como el asma o la dermatitis atópica y la vacunación interfiere este beneficio, 4) no se informa de todas las reacciones adversas provocadas por las vacunas, 5) las enfermedades contra las que vacunamos han desaparecido y hay alternativas homeopáticas para tratar los posibles efectos adversos de las vacunas, 6) la política de vacunaciones está motivada por los beneficios económicos de las industrias farmacéuticas, 7) la vacunación constituye una violación de los derechos civiles y, 8) en las vacunas se usan tejidos provenientes de embriones humanos.

\footnotetext{
${ }^{1}$ Estos argumentos están tomados del trabajo de Wolfe, Sharp y Lipsky (2002) que analizan el contenido y diseño de los sitios web de los distintos grupos antivacunas. La proporción en la que algunos de estos argumentos estaban presentes era: las vacunas causan enfermedades idiopáticas ( $100 \%$ de los sitios), no se informa de todas las reacciones adversas (95\%), la política de vacunaciones está motivada por los beneficios (91\%) (Wolfe et al., 2002, 3245).
} 
Con fines meramente analíticos podemos diferenciar tres aspectos de la controversia:

a. La preocupación de los padres y los grupos antivacunas se centra en los presuntos efectos secundarios que son ampliados y magnificados. Esta es una cuestión de decisión bajo riesgo y la estrategia suele ser un análisis coste beneficio. Las autoridades reconocen que las vacunas pueden tener efectos adversos negativos, por ejemplo encefalitis (1 entre 1 millón) pero la probabilidad de ocurrencia del efecto negativo, frente a los beneficios que reporta, justifica la vacunación. Para los antivacunas esta justificación no es válida o bien porque difieren en la métrica del riesgo, (la probablidad de efectos adversos es mucho mayor de lo que las autoridades sanitarias dicen) o difieren en la concepción del riesgo mismo. No podemos hablar de riesgo en términos de coste beneficio, el riesgo no es una medida objetiva, está mediado por factores sociales y culturales. "Los padres/madres perciben un riesgo individual de enfermar bajo y un balance invertido del beneficio/riesgo de la vacunación, mientras que algunos profesionales opinan que el paciente no debe gestionar este riesgo debido a que la interpretación del mismo precisa conocimiento especializado" (Martínez et al., 2014, 377). Aquí, los actores fundamentes son los padres.

b. Un segundo conjunto de preocupaciones se centra en el papel activo de las vacunas en causar enfermedades cuyo vínculo no está reconocido por las autoridades médicas (autismo como consecuencia de la triple vírica, daños neurológicos por la vacuna de la tos ferina o crisis convulsivas después de la vacuna del VPH). En este caso la discusión se centra en las relaciones causa efecto, cuáles son los requisitos para atribuir causalidad, que se considera evidencia válida, quién tiene la carga de la prueba, etc. Aquí el papel más activo le corresponde a los grupos de expertos que asesoran o forman parte de los grupos contrarios a la inmunización, que discuten con los expertos de la comunidad médica.

c. Un tercer enfoque se centra en las propuestas de los grupos antivacunas, por ejemplo cuando se sostiene que es mejor la inmunización que se produce por vías naturales, es decir, contrayendo la enfermedad; las enfermedades han desaparecido y por tanto ya no es necesario vacunar; las enfermedades contra las que vacunamos no son tan graves; las mejoras en la dieta y la higiene hace a las vacunas innecesarias o existen terapias alternativas a las propuestas médicas al uso. Aquí 
entran cuestiones como qué significa que una enfermedad está erradicada, el nivel de gravedad de las enfermedades contra las que vacunamos. También entran aquí otras concepciones acerca de la medicina: homeopatía y naturopatía frente a la medicina ortodoxa ${ }^{2}$, que a su vez suponen concepciones metodológicas distintas en lo que se refiere al tratamiento de las enfermedades. El enfoque de la medicina ortodoxa es analítico mientras la naturopatía o la homeopatía se basan en "enfoques holísticos" del bienestar y la enfermedad.

En las páginas que siguen se aborda primer lugar, una breve exposición del marco teórico desde el que se realiza el estudio: las controversias científico tecnológicas públicas, para pasar a continuación a un estudio de caso: la muerte en el año 2015 de un niño de 6 años a consecuencia de la difteria. El niño no estaba vacunado por decisión expresa de sus padres. El estudio del caso permite abordar el análisis de las características de algunos de los grupos implicados en la controversia y de distintos aspectos de la misma: las cosmovisiones del mundo sostenidas por los grupos antivacunas, el equilibrio de derechos, el papel de la evidencia, el riesgo o los valores mantenidos. La conclusión que emerge de este estudio es que es precisamente ese conjunto de valores, unido a las cosmovisiones mantenidas, las que motivan diferentes interpretaciones de la evidencia, de la ley, del riesgo y de la ciencia.

\section{Metodología}

La polémica acerca de las vacunas se aborda desde el marco teórico de las controversias científico-tecnológicas públicas (CCTP). Estas se caracterizan por un desacuerdo acerca de un producto o teoría científica o tecnológica en el que interviene distintos expertos. Pero, sobre todo, se caracteriza por la presencia de componentes políticos, ideológicos o morales que influyen en los juicios de los expertos y de la ciudadanía. Ello provoca que la controversia continúe incluso cuando hay un consenso amplio acerca de la evidencia disponible. Ejemplos típicos de controversia son: la energía nuclear, la disputa entre creacionistas y darwinistas, la controversia sobre la relación entre cáncer y dieta o la controversia sobre la maternidad subrogada ${ }^{3}$.

\footnotetext{
${ }^{2}$ Se utiliza medicina ortodoxa como aquella conforme a hábitos o prácticas generalmente admitidos frente a la heterodoxia de las medicinas o terapias alternativas.

${ }^{3}$ Estos son algunos de los casos de estudio que aparecen en el clásico de D. Nelkin (1992). Cada uno de los casos ilustra un tipo de controversia: las que se encuadran entre prioridades políticas y valores ambientales, las que se producen entre derechos individuales y fines sociales, las que oponen intereses económicos al control de riesgos para la salud y en las que se enfrentan sentimientos morales a los valores
} 
Dos rasgos fundamentales en este tipo de disputas son; por un lado, que los resultados experimentales son desacreditados o reinterpretados para adecuarse a cada parte de la contienda y que los datos son usados selectivamente para apoyar una posición u otra. Por otro lado, en las disputas abundan los mecanismos retóricos. Los más frecuentes son: «no hay evidencia que muestre que ... pero», la desacreditación del contrario como experto, la carga de la prueba y el discurso coste beneficio (Mazur, 1981). Cada uno de estos recursos está presente en el debate acerca de las vacunas. Por ejemplo, los grupos antivacunas hablan de los efectos a largo plazo de las vacunaciones en el organismo, tales efectos no han sido investigados y, por tanto, según ellos no se puede descartar que las vacunas tengan efectos adversos a largo plazo. Esto supone un cambio de la carga de la prueba, dado que el que argumenta la existencia de un efecto debe aportar las pruebas necesarias para su contrastación. En cuanto al discurso coste beneficio se sabe que las vacunas, como cualquier otro medicamento, pueden tener reacciones adversas que están además tipificadas y estimadas probabilísticamente en los prospectos de las mismas. Por parte de las autoridades médicas se afirma que el riesgo de sufrir una reacción adversa de gravedad es tan bajo, que los beneficios de vacunar a la población exceden con creces los riesgos. Pero como se verá más adelante, el riesgo o la percepción del mismo no es un factor objetivamente mensurable, las percepciones acerca del riesgo son influenciadas por un rango de factores políticos, culturales e institucionales.

Contrariamente a las posiciones más ortodoxas, parece que la evidencia tiene poco peso en la dinámica y resolución de las controversias científico-tecnológicas. Esto es así por varios factores: en primer lugar, la evidencia es solo una de las cuestiones implicadas, incluso cuando no hay desacuerdo acerca de la misma, los desacuerdos políticos o ideológicos pueden continuar. En segundo lugar, se puede afirmar que a veces los expertos no discrepan sino hablan de cuestiones completamente distintas, la cuestión de qué es un error y qué es lo correcto depende de lo que está siendo calculado, en general no hay un acuerdo acerca de qué constituye evidencia relevante.

Finalmente, la evidencia siempre puede ser desafiada. Algunos autores hablan de que existe una infradeterminación radical de la teoría por los datos, es decir, disponemos de evidencia que avala dos hipótesis contradictorias, por tanto, la evidencia no nos permite elegir entre ellas y no contribuye a saldar la controversia. Incluso cuando no se sostienen tesis radicales de infradeterminación, una gran parte de las controversias se producen en el marco de lo que 
es ciencia regulativa, es decir, la ciencia que se utiliza como base para la toma de decisiones políticas (Jasanoff, 1995) y este tipo de ciencia presenta una cierta infradeterminación. Veamos en qué sentido puede esto ser afirmado sin caer en las tesis más radicales.

Tal como plantea Mazur (1981) cuando es necesario hacer un supuesto simplificador ¿qué simplificación puede ser hecha? Cuando no hay datos acerca de una cuestión ¿cuántos datos pueden ser razonablemente extrapolados de otra cuestión? ¿Cómo de confiable es un conjunto de observaciones empíricas? Un ejemplo típico es la extrapolación "dosis-respuesta" que se realiza en la evaluación de un posible agente cancerígeno. Es imposible detectar los efectos tóxicos de un agente a dosis bajas, por lo que los estudios se realizan a dosis altas que después son extrapolados. A falta de datos se supone que esta curva es lineal, pero también puede ser logarítmica.

Gran parte de las controversias surgen en un contexto de ciencia regulativa (Jasanoff, 1987), es decir, aquella ciencia que sirve como fundamento en las decisiones políticas de regulación. La ciencia regulativa posee algunas características propias: en primer lugar, es un terreno propicio a interpretaciones conflictivas de sus resultados y esas interpretaciones están socialmente determinadas. Frente a la ciencia que se desarrolla en laboratorios y universidades la ciencia regulativa se realiza en las instituciones de la industria y el gobierno. Es una ciencia por mandato, frente a la relativa libertad de investigación de la ciencia académica. Ello tiene que ver con los plazos, es decir, los científicos han de presentar resultados en un plazo determinado de tiempo frente a la ausencia de plazos estrictos de la segunda. Como consecuencia de la anterior, es posible que se tenga que tomar una decisión antes de que todos los datos estén disponibles, lo cual a su vez repercute en las posibilidades de alcanzar un consenso. Siempre existe la posibilidad de esperar por más datos, pero como señala Jasanoff esto supone un trade-off entre ciencia y seguridad. Tal vez la mayor diferencia radica en los procedimientos de evaluación: frente a la revisión por pares propia de la ciencia académica la ciencia regulativa es evaluada en procesos judiciales o políticos y cuando el peer review se utiliza en contextos regulativos surgen desacuerdos acerca de lo que debe ser evaluado. Por ejemplo: “¿deben las agencias enviar los protocolos de los nuevos tests o las guías de interpretación para revisar o solo estudios completos? ¿Deben las evaluaciones de riesgo ser revisadas o solo los datos primarios sobre los que están basadas las evaluaciones?" (Jasanoff, 1987, 218). Pero tal vez la principal característica de la ciencia regulativa, como ya se señaló más arriba, es la infradeterminación. 
Una cuestión fundamental en este tema es el análisis de los factores que inciden en el cierre de una controversia. Brian Martin señala algunos elementos claves $(2014,113)$ : (a) Los principales partidarios de una de las facciones mueren o se retiran. (b) Las revistas científicas y los medios de comunicación dejan de cubrir el debate. (c) Científicos, políticos o editores, que constituyen la opinión informada, se decantan por uno de los lados y son seguidos por el resto de los participantes. (d) Uno de los lados está tan estigmatizado que pocos apoyan su posición (e) Todas las fuentes de financiación de la investigación aceptan una de las posiciones por lo que es imposible investigar alternativas. (f) Los detractores del establishment son discriminados llegando al extremo de perder sus puestos de trabajo. (g) Un gobierno toma una decisión que respalda a uno de los contendientes y adopta políticas para hacerla cumplir. (h) Un referéndum público apoya uno de los lados.

Ernan McMullin (1987) distingue tres tipos distintos de clausura de una controversia:

1. Resolución. Donde la controversia se resuelve sobre la base de factores epistémicos aceptados por ambos contendientes. En un sentido ideal ello implicaría que la comunidad comparte las reglas de evidencia e inferencia, es decir qué es lo que cuenta como evidencia satisfactoria para resolver la controversia y que tal evidencia ha sido descubierta. Sería el punto c señalado por Martin.

2. Clausura. Donde predominan los factores no epistémicos, especialmente aquellas situaciones en las que una autoridad externa declara una controversia finalizada. Los puntos e, $\mathrm{g} \mathrm{y} \mathrm{h}$ de Martin.

3. Abandono. En general se produce cuando los contendientes pierden interés en la disputa y esta no es resuelta pero tampoco permanece activa. Los puntos a y $b$ de Martin.

\section{El caso}

En junio de 2015 fallece en Olot (Cataluña) un niño de 6 años a consecuencia de la difteria, no estaba vacunado por decisión expresa de sus padres a pesar de que la vacuna entra en el calendario de vacunación gratuito en España ${ }^{4}$. Tras el fallecimiento, 47 niños del mismo pueblo (1.5\% de los menores del municipio) continuaban sin ser vacunados ${ }^{5}$.

\footnotetext{
${ }^{4}$ En España la vacunación contra la difteria se inició en 1945, la primera campaña nacional masiva fue en 1965. (Instituto de Salud Carlos III, s.f., 27)

${ }^{5} \mathrm{http} / / /$ xurl.es/efd7j. Consultado el 15/06/2018
} 
Claves para comprender la resistencia de los colectivos antivacunas: / Obdulia Torres González / 15 una controversia científico-tecnológica pública

En mitad de la crisis, la Liga para la libertad de vacunación un conocido grupo que aboga por la eliminación de las vacunas, emite un comunicado de prensa en el que afirma:

Desde la Liga por la Libertad de Vacunación llamamos a las familias a continuar ejerciendo su derecho a decidir informada y libremente sobre la vacunación, reafirmando toda decisión tomada responsablemente, y exhortamos a las autoridades sanitarias, ante la actual carencia de datos certeros sobre el origen de este único caso de manifestación de la enfermedad, a que hagan un análisis contextualizado y correcto de la situación ${ }^{6}$.

También la Asociación de afectados por el virus del papiloma humano (AAVP) se pronuncia sobre el tema:

Aprovechar lo ocurrido para querer obligar a los padres a administrar sea cual fuere un medicamento, en una situación sanitaria como la de nuestro país, no puede más que obedecer o a la ignorancia o a intereses más vinculados a lo económico que a lo propiamente sanitario. La AAVP, lamenta profundamente que se quiera utilizar un hecho tan lamentable como lo que le ha ocurrido al niño de Olot, para querer obligar a los padres a vacunar a sus hijos/as, sobre todo si tenemos en cuenta el desamparo absoluto en el que se encuentran las victimas cuando tienen la desgracia de sufrir reacciones adversas por una vacuna. Desde la AAVP esperamos que impere el sentido común, que se deje ya de criminalizar a los padres del niño de Olot y que cejen ya las amenazas de quienes quieren imponer su criterio, incluso expedientando a los expertos que no lo compartan ${ }^{7}$.

Por su parte los padres del niño declaran sentirse engañados por los antivacunas ${ }^{8}$.

A su vez el Conseller de Salud de la Generalitat de Cataluña se propone difundir información "veraz" sobre las vacunas al conjunto de la ciudadanía".

\footnotetext{
${ }^{6} \mathrm{http}: / /$ xurl.es/duu0w. Consultado el 15/06/2018

${ }^{7}$ http://xurl.es/hy76e. Consultado el 15/06/2018

8 “El director general de la Agència de Salut Pública, Antoni Mateu, ha explicado que los padres del niño de 6 años de Olot infectado de difteria están 'absolutamente destrozados' y se sienten 'engañados' por la información proporcionada por los grupos antivacunas. Los progenitores también se sienten 'culpables' por haber confiado en ellos". Cita de El Periódico, 5 de junio de 2015. http://xurl.es/jqeks Acceso el $15 / 10 / 2017$

${ }^{9} \mathrm{http}: / /$ xurl.es/cw5wc
} 


\section{Los actores implicados}

Normalmente en la dinámica de las controversias suelen existir distintos grupos de actores con roles determinados. Una lista no exhaustiva incluiría: políticos que piden asesoramiento a científicos expertos en alguna tecnología; grupos de afectados por esa tecnología que a su vez son representados por organizaciones ciudadanas, de derechos, ecologistas, ... que a su vez contratan científicos expertos; industrias, que representan los intereses de desarrollo de la tecnología que a su vez pueden tener sus propios científicos expertos.

Los actores políticos están representados en este caso por el Ministerio de Sanidad. Más concretamente el Conseller de Salud de la Generalitat, Boi Ruiz que pone sobre la mesa la obligatoriedad de las vacunas a raíz de la muerte del niño de Olot. Según la prensa del momento el Conseller "[s]e ha vuelto a mostrar partidario de la obligatoriedad, y ha dicho que, si fracasa la etapa del «convencimiento», será necesario abrir el debate sobre la obligación de vacunar"10 $^{\prime 10}$ En España la vacunación es voluntaria, solo puede ser impuesta por las autoridades sanitarias cuando exista un riesgo para la salud pública.

Las actitudes del público respecto a las vacunas varían mucho: según Elizabeth Miller ${ }^{11}$ los investigadores han identificado cinco actitudes distintas de los padres que representan un continuo respecto a la vacunación.

En primer lugar, los de aceptación incondicional (30-40\%), quienes creen en los beneficios de la vacunación o confían en los consejos de su médico. En segundo, los que lo aceptan con cautela (25-35\%), que tienen algunas preocupaciones menores, pero realizan la vacunación tras una breve discusión sobre efectos secundarios y riesgo de enfermedad. En tercer lugar, el grupo indeciso (20-30\%), que engloba a aquellos que tienen preocupaciones significativas sobre el riesgo de las vacunas. Para este grupo es clave la información proporcionada por médicos y enfermeras, si sus dudas son solucionadas por personal médico competente procederán a vacunar. En cuarto, los que mantienen una vacunación selectiva (2-27\%), que tiene preocupaciones específicas acerca de una vacuna o de un fenómeno concreto como sobrecarga inmunológica. Y, finalmente, (menos de 2\%) los que rehúsan de forma rotunda todas las vacunas motivados por creencias religiosas, filosóficas o alternativas. Este grupo es improbable que cambie de actitud. (Miller, 2015, 3)

\footnotetext{
${ }^{10} \mathrm{http}: / /$ xurl.es/nb7x4

${ }^{11}$ Elizabeth Miller es Consultant Epidemiologist en the Immunisation Hepatitis and Blood Safety Department,Public Health England (PHE) in Colindale North, West London
} 
Dentro de este último grupo que rechaza radicalmente las vacunas tenemos las comunidades de neorrulares que tienen, además, un fuerte asentamiento en la comarca de la Garrotxa, donde se encuentra el municipio de Olot, y de hecho fueron el blanco de algunas informaciones de la prensa.

Por neorrural se entiende "el fenómeno de instalación en el campo de un colectivo mayoritariamente joven y procedente de zonas urbanas [...] que abandonan la ciudad y se dirigen al campo con un proyecto de vida alternativo" (Nogué i Font, 1988, 145). Es importante señalar que ese retorno al campo tiene un enorme valor simbólico como reencuentro espiritual del ser humano con la naturaleza, hasta el extremo que se les denomina los inmigrantes de la utopía (Herviu y Leger, 1979; Nogué, 1988). Los neorrulares reaccionan contra un modelo de sociedad, de relaciones económicas e interpersonales, denostan la ciudad, siendo el campo y la naturaleza su utopía, de ahí una concepción de la naturaleza y lo natural como esencialmente bondadoso, frente a la maldad intrínseca de todos aquellos productos resultado de la intervención humana. Sus influencias históricas se sitúan en la tradición del pensamiento utópico y más recientemente con el movimiento contracultural de Europa y América en los años 60, con el romanticismo de los siglos XVIII y XIX en lo que refiere a la relación del hombre con la naturaleza como algo bello para disfrutar y no para sacarle provecho y en el ecologismo (Ibargüen et al., 2004). Las motivaciones para ese cambio radical de vida tienen que ver fundamentalmente con la coherencia de un conjunto de valores que rigen su vida. Valores que fundamentalmente rechazan lo material en favor de una vida más espiritual y que reivindica la cultura y los oficios tradicionales. "Lo salvaje, lo natural se identifica con lo auténtico" (Ibargüen, 2004, 15). En la misma provincia, Girona, tiene su sede la asociación denominada Liga para la Libertad de la Vacunación. Es una asociación sin ánimo de lucro fundada en 1989. Entre sus objetivos destaca: que la medicina pública oferte otras opciones distintas a lo que ellos denominan medicina alopática (homeopatía, naturismo) y que se respeten las decisiones individuales respecto a la conveniencia o no de vacunar a sus hijos. Otro conjunto de reivindicaciones se refiere a la farmacovigilancia de las vacunas y la respuesta de la administración ante posibles reacciones adversas ${ }^{12}$. Está compuesta, según su página web, por padres y profesionales de la salud, lo que hace que cuenten con su propio grupo de expertos quienes organizan conferencias, cursos y actividades de difusión en toda España. Ambos colectivos, tanto los neorrurales como la Liga formarían parte de ese dos por ciento absolutamente refractario a cualquier

${ }^{12}$ Los objetivos y demandas están tomados de su página web http://xurl.es/1nmvn. Consultado el $25 / 02 / 2018$ 
tipo de vacuna, pero su influencia sobre el colectivo que más duda presenta es enorme debido a que la fuente principal de información a la que recurren los padres en caso de dudas acerca de las vacunas suele ser Internet donde tienen una fuerte presencia.

Los grupos de expertos que asesoran a la administración se agrupan fundamentalmente, en el comité asesor de vacunas, este comité es el portavoz oficial de la Asociación Española de Pediatría en todo lo que tiene que ver con vacunas. Está constituido, según su página web, por pediatras de reconocido prestigio en el campo de la infectología y vacunología. Un segundo grupo de actores es la Asociación Española de Vacunología, una asociación científica sin ánimo de lucro, cuya misión declarada según su página web es proteger a las personas y mejorar la salud de la población, fomentando el uso idóneo de las vacunas para la prevención de enfermedades infecciosas. Su web está acreditada por la red de seguridad vacunal de la OMS. Pero entre sus patrocinadores se encuentran Sanofi Pasteur, Pfizer y Baxter, tres grandes industrias farmacéuticas ${ }^{13}$, lo que pone en el punto de mira los intereses comerciales de las farmacéuticas y elimina su credibilidad de cara a los grupos antivacunas. Analizado desde la perspectiva del caso de Olot, la cuestión fundamental es el rechazo a la vacunación por motivos ideológicos y la reivindicación de la libertad de elección.

Este es solo uno de los escenarios de la controversia de las vacunas en España. Otro se produjo en febrero de 2009 cuando dos niñas de 14 años fueron ingresadas con un cuadro de convulsiones y pérdida de conocimiento tras haber sido vacunadas contra el virus del papiloma humano. El comité de expertos de la agencia española del medicamento publica una nota informativa en la que concluye que "la ausencia de una base biológica que explique la posible asociación entre los trastornos paroxísticos presentados y las vacunas hace muy improbable que dichos cuadros puedan considerarse como reacciones adversas a las mismas"14. Pese a esto la controversia continua en los medios y se funda la Asociación de afectadas por la vacuna del papiloma ${ }^{15}$ que piden el reconocimiento de los efectos adversos y un fondo de compensación

\footnotetext{
${ }^{13}$ En su página web la Asociación afirma: “[1]as entidades participantes patrocinan económicamente el mantenimiento de este Web sin participar en su contenido ni en su línea editorial, lo cual es decisión única y exclusiva del Comité Editorial de la misma y bajo supervisión de la Junta Directiva de la AEV'. Pero la presencia de estos patrocinadores alimenta los argumentos de sus oponentes acerca de la relación entre los beneficios económicos de las industrias farmacéuticas y los calendarios de vacunación.

14 Comunicación sobre riesgos de medicamentos para profesionales sanitarios. Ref: 2009/06 23 de abril de 2009. Nota informativa. Seguridad de las vacunas frente al virus del papiloma humano: conclusiones del comité de expertos. En http://xurl.es/pqd7q. Consultado el 27/06/2018

${ }^{15} \mathrm{https}$ //asociacion.aavp.es
} 
para los afectados por las vacunas. Otro actor importante en este escenario es la Asociación de afectados por las vacunas con los mismos objetivos. En este escenario la controversia gira en torno al riesgo, la percepción del mismo y el análisis coste beneficio.

Estos dos escenarios dan entrada a algunos de los aspectos en discusión. La controversia sobre las vacunas es una controversia poliédrica que presente muchas facetas distintas: (1) cuestiones relativas al equilibrio de derechos, la cuestión es qué derechos deben prevalecer, si los de los grupos que se oponen a la vacunación como una cuestión de libertad de elección de tratamiento médico o los que favorecen la vacunación y ven que la inmunidad de grupo peligra. (2) Cuestiones relativas a lo que se considera ciencia. Los grupos opuestos a la vacunación recurren a terapias y tratamientos alternativos como homeopatía la en el tratamiento de los efectos adversos de las vacunas que son considerados pseudocientíficos por los provacunas. (3) Diferentes percepciones del riesgo por parte de los actores implicados. Más allá de los estudios psicométricos, en los colectivos antivacunas parecen estar operando valores y factores culturales a la hora de determinar la percepción del riesgo de cada grupo en el sentido planteado por Mary Douglas (Douglas y Wildavsky, 1982). (4) Diferentes concepciones acerca de la experticia científica y de quién es un experto científico. (5) Distintas cosmovisiones que engloban la relación del cuerpo humano con su entorno y la visión de la naturaleza. (5) Diferentes concepciones de la evidencia.

\section{Valores y cosmovisión del mundo}

En un barrio de Granada (España) se produjo en el año 2010 un brote de sarampión que finalizó con una decisión judicial, en la que se obligaba a los menores del entorno en el que había surgido el brote a ser vacunados. Un año después, un grupo de investigadores realizó un estudio en el que analizaron, a través de entrevistas semiestructuradas, el discurso de aquellos padres que decidían no vacunar a sus hijos. A continuación, se transcriben algunos de los fragmentos de los entrevistados: «Prefiero que mis hijos, y yo misma, pasemos el sarampión u otras enfermedades de manera natural, sin arriesgar sus vidas poniéndole la triple vírica». «Mantener una salud óptima requiere que los niños sean felices y se diviertan, que tengan buenos pensamientos y buena salud emocional, alimentación natural, aire fresco, luz natural, ejercicios... es la condición del cuerpo la que permite o no la enfermedad» «Saludable es lo que respeta lo natural... sin agresiones, sin tóxicos... sin hacer daño a la especie humana...» (Martínez et al., 2013). Es llamativo en este 
discurso la continua apelación a lo natural o a la Naturaleza. Es una falacia argumentativa en el sentido de que identifica lo natural con lo bueno y lo artificial con lo malo. Pero más allá del error de argumentación subyace aquí una auténtica cosmovisión del mundo. Más arriba se señalaba, en relación a los grupos neorrurales, el valor simbólico del reencuentro espiritual del ser humano con la Naturaleza, así como el rechazo de lo material y lo artificial. Rogers y Pilgrim (1995) asocian el rechazo radical a las vacunas con grupos que sostienen creencias New Age, las cuales coinciden en gran medida con las de estos grupos de neorrurales. Estas cosmovisiones engloban intereses tan diversos como:

[...] la ciencia moderna, las filosofías orientales, la psicología del potencial humano, las religiones de los indígenas norteamericanos, la mitología de la diosa y el matriarcado primitivos, las terapias que integran cuerpo y mente y todo aquello que se considera natural (como por ejemplo la comida orgánica, la sanación natural y la ecología. (Carozzi, 1993, 19)

La New Age es un conjunto de creencias, valores y prácticas sumamente eclécticas y no siempre consistentes. Para el tema que nos ocupa hay que resaltar, en primer lugar, la ya mencionada relación íntima de la especie humana con la naturaleza. Es la rotura de ese vínculo la responsable de gran parte de los males que aquejan al mundo, y el origen de esos problemas es la visión ilustrada del conocimiento, la ciencia y la tecnología (Carozzi, 1993, 22). En segundo lugar, es especialmente relevante la creencia en la unidad de materia, mente y espíritu en los seres humanos, donde cada uno de los planos puede afectar al resto, la sanación se entiende por tanto en un sentido holista (opuesto a mecanicista) y entran aquí en juego un enorme número de terapias de sanación alternativas. Estas terapias se caracterizan por:

[...] considerar a la persona como un todo en continua interacción y cambio con el entorno, integrando aspectos físicos, espirituales, mentales, emocionales, genéticos, medioambientales y sociales [...] tanto la naturopatía como la homeopatía tienen como objetivo estimular la capacidad del cuerpo de curarse a través de cambios en la nutrición y el estilo de vida. (Ministerio de Sanidad, 2011, 5)

Esto coincide con los resultados de los estudios acerca de las razones de los padres para no vacunar, ya que muchos de ellos piensan que si sus hijos tienen dietas y estilos de vida saludables corren mucho menor riesgo de contraer las enfermedades infantiles para las que se vacuna (McKee y Bohannon, 2016). 


\section{El equilibrio de derechos}

Un tema sumamente controvertido es la prevalencia de los derechos de uno u otro grupo. La vacunación no es obligatoria en España, pese a eso los denominados grupos antivacunas se sienten presionados por el entorno social, escolar y sanitario a vacunar a sus hijos. Estos grupos hacen especial hincapié en los derechos que les otorga la Constitución Española. En concreto invocan el artículo 15 que reza: "Toda persona tiene derecho a la vida y a la integridad física y moral, sin que, en ningún caso, pueda ser sometida a tortura ni a penas o tratos degradantes". Al invocar este artículo equiparan la vacunación a la tortura. A su vez el artículo 10.9 de la Ley General de Sanidad afirma que todos tienen derecho a negarse a un tratamiento excepto cuando esta negativa suponga riesgo para la salud pública, no se esté capacitado para tomar decisiones, o cuando la urgencia no permita demoras por poderse causar lesiones irreversibles o peligro de fallecimiento. Por otro lado, el artículo 28 establece que no se podrán tomar medidas obligatorias, por parte de las autoridades sanitarias, que conlleven riesgo para la vida. La aplicación de estos principios depende precisamente de lo que se considere riesgo para la salud pública y riesgo para la vida. Son precisamente estos dos riesgos los controvertidos en el tema que nos ocupa, por lo que la controversia se extiende al ámbito legal sin posibilidad de resolución.

Más allá de la cuestión legal existe una cuestión moral entre el derecho de cada individuo a elegir la forma en que quiere desarrollar su propia vida, y por tanto negarse a que a sus hijos se les administre un tratamiento que consideran potencialmente peligroso, y el derecho del resto de la comunidad a que sus hijos disfruten de un entorno sano, derecho que a su vez es garantizado por ley. El artículo 3 de la Ley de medidas especiales en materia de Salud Pública establece:

[...] con el fin de controlar las enfermedades transmisibles, la autoridad sanitaria, además de realizar las acciones preventivas generales, podrá adoptar las medidas oportunas para el control de los enfermos, de las personas que estén o hayan estado en contacto con los mismos y del medio ambiente inmediato, así como las que se consideren necesarias en caso de riesgo de carácter transmisible.

Esta fue la ley invocada en relación al caso de Granada que se citaba más arriba. El juez obligó a vacunar a 35 niños ante un brote de sarampión que ya afectaba a 46 personas, 14 de las cuales habían necesitado atención hospitalaria. Entre las consideraciones esgrimidas por el magistrado estaba que la enfermedad se estaba cebando en dos grupos de riesgo, bebés de menos de 
15 meses, edad a la que en ese momento se administraba la vacuna (de los 46 casos registrados 17 eran bebes) y personas de entre 25 y 40 años que no sufrieron la enfermedad en su infancia, pues en ese momento había experimentado un descenso respecto a décadas anteriores y no fueron vacunados dado que en ese momento no existían las vacunas ${ }^{16}$.

En ese conflicto insoluble de derechos hay un factor adicional y es que los antivacunas son vistos como free riders o gorrones (Miller, 2015) que rehúsan vacunar a sus hijos en la convicción de que estos están seguros debido a la inmunidad de grupo. En un estudio llevado a cabo en 1996 acerca de la actitud de los padres hacia la vacuna se encontró que cuando se les preguntó a los padres qué harían si todo el resto de los niños estuvieran vacunado el 94\% de los que el estudio consideraba antivacunas y el 5\% de los considerados provacunas afirmó que no vacunaría a sus hijos (Meszaros et al., 1996, 701). Este hecho refuerza la opinión negativa sobre los antivacunas en algunos sectores sociales.

En una encuesta del periódico La Vanguardia un 91.4\% de los encuestados (3270 personas) opinaban que las colonias deberían vetar a los niños sin vacunar $^{17}$. Lo que supone primar los derechos del colectivo frente a los derechos individuales de elección. En ese sentido hay quien apela a la reestructuración de la discusión sobre la vacunación considerándolo no como una elección individual sino como un bien social (Leach y Fairhead, 2007, 16).

\section{Las controversias y las ciencias de frontera}

Ya se vio como dentro de los objetivos de la Liga para la Libertad de Vacunación figuraba la petición de que la medicina pública oferte tratamientos médicos alternativos (naturopatía y homeopatía) a la medicina ortodoxa, abriendo así un nuevo frente de batalla entre provacunas y antivacunas. En este caso la pugna se centra en aquello que puede ser considerado conocimiento científico y por tanto que cuenta como evidencia. Por ejemplo, los homeópatas pretenden ser evaluados de forma diferente a los fármacos convencionales:

No podemos ajustarnos a un marco conceptual de tipo mecanicista y pensado para operar bajo el paradigma de la teoría molecular [...] la homeo-

\footnotetext{
${ }^{16}$ El País http://xurl.es/dsadd consultado el 29/07/2016. La vacuna contra el sarampión se introduce en España en 1978.

${ }^{17}$ El niño fallecido en Olot había ido de colonias en los días anteriores al desarrollo de la enfermedad. http://xurl.es/4ryya Consultado el 29/07/2016
} 
patía nunca va a poder demostrar su cientificidad en estas condiciones. Por ejemplo, aceptar el doble ciego en las condiciones en que se hace habitualmente, es un imposible para la homeopatía, puesto que la acción del remedio no es universal, sino que está sujeta a la noción de susceptibilidad. (Mora, 2008, 10)

Bajo estas condiciones la medicina y farmacología ortodoxa declara a la homeopatía una pseudociencia.

Thomas Gieryn habla del trabajo de frontera ${ }^{18}$ a la hora de delimitar lo que es ciencia de lo que no lo es. Las cuestiones importantes aquí son “¿Dónde está la frontera entre ciencia y no ciencia? ¿Qué argumentos o prácticas son científicas? ¿Quién es un científico? ¿Qué es la ciencia?” (1995, 393). Una vez delimitado el conocimiento científico todos aquellos que permanecen fuera pierden la legitimidad para entrar en la discusión. Por eso los intentos, en muchas de las páginas web, de invocar la cientificidad de las terapias alternativas como la homeopatía. Pero aquí los universos de discurso se separan radicalmente. Tal vez sea este uno de los mejores ejemplos de la inconmensurabilidad kuhniana cuando afirmaba que los miembros de los distintos paradigmas habitan mundos distintos. La cita anterior acerca de la cientificidad de la homeopatía pertenece a Madeleine Bastide, una profesora de inmunología y parasitología de la Facultad de Farmacia de la Universidad de Montpellier, en la entrevista citada y preguntada acerca de los posibles efectos adversos de la vacuna de la hepatitis B afirmaba:

Los alópatas no ven nada y todas las observaciones provinieron de homeópatas y osteópatas. No es la misma semiología.

Entrevistador-Es una cuestión de la preparación del observador. M.B.-Exactamente.

Entrevistador-A veces la dificultad estriba en el hecho de relacionar enfermedades que a priori no tienen nada que ver con la vacuna inoculada.

M.B.-En nuestro estudio de la Hepatitis B, el único efecto secundario que los alópatas estaban preparados para ver era la Esclerosis Múltiple, porque es la idea que tienen, porque ya entra en sus esquemas como un posible

\footnotetext{
${ }^{18} \mathrm{La}$ frontera se refiere a la línea que separa la ciencia de la política. Las concepciones más positivistas de la ciencia sostienen que esa frontera es nítida y que las cuestiones políticas, los factores externos, quedan fuera de la ciencia. En la actualidad se sostiene que esa frontera es un territorio borroso en el que muchas cuestiones científicas y políticas se entremezclan. La frontera puede ser entendida como transciencia, en la formulación de A. Weinberg (1974), ciencia regulativa de acuerdo con Jassanof (1987) o cuestiones de demarcación para T. Gieryn (1995).
} 
efecto secundario de la Hepatitis B. Pero cuando hay un Guillem-Barré no lo ven. (Mora, 2008, 12)

La autora habla de la farmacología informacional frente a la farmacología molecular clásica presentándola como un nuevo paradigma. Es el marco teórico el que determina que observaciones son relevantes, por tanto, pro y antivacunas observan cosas distintas cuando tratan a los mismos pacientes. Asimismo, el paradigma determina cuál es la noción de prueba aceptable y qué métodos nos permiten validar el conocimiento. En este sentido, la posición epistemológica kuhniana les permite presentarse como un nuevo paradigma emergente y luchar por la legitimidad de su discurso.

\section{La estructura epistémica de la situación}

Mary Morgan expone las dificultades epistémicas y cognitivas que se presentan a la hora de persuadir a los padres acerca de algún aspecto de la salud de sus hijos de las que ellos tienen experiencia observada. La tesis de Morgan es que las observaciones que provienen de la experiencia personal no pueden ser tan fácilmente descartadas en ciencias sociales como lo son en ciencias naturales, a pesar de ello las posibilidades de expresar esa experiencia dependen en parte de la ciencia social implicada y de la epistemología cívica del ambiente en el que son expresadas. Analiza el caso de la vacuna triple vírica como un ejemplo a caballo entre las ciencias sociales y las naturales. La pregunta a la que pretende dar respuesta Morgan es por qué un médico no puede decir a un padre que el autismo de su hijo es resultado de la vacuna triple vírica. En "Voice and the facts and observations of experience" (2010) expone los factores epistémicos que inciden en el rechazo de la información por parte de los padres.

En primer lugar, señala Morgan, la experiencia estadística afirma que la conexión entre la vacuna y la enfermedad es altamente improbable, pero ello no refuta necesariamente el hecho de que, en algunos casos, dada la variabilidad de los factores implicados, no se pueda afirmar tal conexión. El hecho es que la evidencia estadística se aplica a muestras y poblaciones, pero es epistémicamente inadecuada para explicar los casos individuales. Un segundo factor refiere a las diferencias entre el conocimiento del médico, basado en la práctica clínica y el estudio de caso por caso, del que obtiene las generalizaciones posteriores y la investigación médica basada en el trabajo estadístico. Morgan extiende el argumento un poco más allá para hablar de la experiencia de los padres, basada en la observación de su hijo y afirma: 
Una madre puede reconocer mejor que su médico los signos de lo que sucedió y cuándo sucedió al observar eventos estrechamente relacionados, porque es su propio hijo, a quien conoce más y ha observado con más cuidado y consistencia de lo que cualquier médico podría haber hecho. (Morgan, 2010, 54)

El tercer factor que estructura esta situación es la falta de respuestas acerca de las causas de la enfermedad. Y finalmente, los cambios que se ha producido en la relación médico paciente, donde el médico ya no goza de la autoridad de la que disfrutaban y los pacientes son considerados como consumidores bien informados que tienen además la capacidad de obtener conocimiento de otras fuentes e interpelar con él al médico.

Los puntos uno y dos son los fundamentales para el tema que nos ocupa. El punto uno se refiere a los problemas de los estudios epidemiológicos ${ }^{19}$ :

- $\quad$ el hecho de que los individuos dentro de la muestra no son idénticos lo que produce errores sistemáticos,

- la promediación que produce artefactos ${ }^{20}$ estadísticos y esta, a su vez la falacia ecológica ${ }^{21}$,

- los problemas de validez interna y externa,

- la creencia de que la randomización estricta de un gran número de sujetos controla todas las formas de sesgo,

- $\quad$ el hecho de que no proporcione una comprensión de los fenómenos bajo estudio, dado que no se ocupa de las causas subyacentes a los fenómenos (Charlton, 1996).

El análisis de la confiabilidad de los métodos estadísticos excede los límites del presente trabajo sólo se quiere hacer constar que la discusión acerca de la validez de los estudios epidemiológicos respecto a las vacunas se encuadra en una controversia científica, o metodológica, que alienta los argumentos de los padres. El punto dos vuelve a remitirnos a los métodos de evaluación de la evidencia: "[m]ientras que la medicina convencional ha adoptado para la evaluación de los procedimientos terapéuticos el patrón oro del ensayo clínico aleatorizado, las terapias naturales han centrado la evidencia de su utilidad

\footnotetext{
${ }^{19}$ Se entiende epidemiología como el estudio de la salud en la población

${ }^{20}$ Una entidad que no se corresponde a ninguna instancia individual y solo es verdadera a nivel del grupo.

${ }^{21}$ La falacia ecológica se produce cuando se infiere la naturaleza de los individuos partiendo de los datos del agregado
} 
en la tradición y la experiencia individual y colectiva de su uso" (Ministerio de Sanidad, 2011, 13).

\section{Diferentes percepciones del riesgo}

La controversia de las vacunas es fundamentalmente una controversia sobre riesgo. La vacuna triple vírica puede causar una encefalitis en una de cada millón de dosis, la del meningococo el síndrome de Guillain-Barré con la misma probabilidad ${ }^{22}$. Por otro lado, en países industrializados la letalidad del sarampión es de 1/10000 casos notificados según la Asociación Española de Pediatría. La estrategia de las autoridades sanitarias en sus comunicados provacunales es comparar la tasa de mortalidad con y sin vacuna. En este caso sólo debemos comparar un millón frente a diez mil. Ello implica que hay una medida objetiva del riesgo y que este es percibido por todos los sujetos por igual. Pero según McKee y Bohannon en su estudio sobre las razones de los padres para no vacunar:

[a]lgunos padres creen que las enfermedades para las que vacunamos no son muy frecuentes, por lo que sus hijos corren un riesgo mínimo de contraer estas enfermedades. Por esta razón, también creen que los posibles efectos secundarios negativos de la administración de la vacuna superan los beneficios de las mismas. Muchos padres no ven las enfermedades prevenibles como graves, o como una amenaza para la vida, y preferirían no poner productos químicos adicionales en los cuerpos de sus hijos. (2016, 107)

Parecen estar operando aquí dos lógicas distintas, en lo que a percepción del riesgo se refiere, y esto es lo que nos confirman las investigaciones en percepción del riesgo. La literatura ofrece tres paradigmas distintos que no solo definen el riesgo de distinta manera, sino que identifican distintas variables que afectan a los individuos en la percepción del mismo. El primero es el modelo axiomático, que coincidiría a grandes rasgos con el modelo que presuponen las autoridades sanitarias. Este modelo combina las consecuencias de las decisiones de riesgo (mortalidad, lesión, pérdidas financieras, etc.) con la probabilidad de su ocurrencia. Los axiomas conductuales en los que se basa son transitividad y monotonicidad. Los estudios empíricos han mostrado violaciones de estos axiomas, debido fundamentalmente a que los sujetos no se comportan igual ante la expectativa de pérdidas que de ganancias (Kahneman

\footnotetext{
${ }^{22}$ Según el Manual de Vacunas en línea de la Asociación Española de Pediatría http://xurl.es/8elbr consultado el 05/07/2018.
} 
y Tversky, 1987). Por otro lado, se han mostrado diferencias interculturales en la percepción del riesgo que son incluso más elevadas que las diferencias entre sujetos con distintas ocupaciones. En segundo lugar, están los estudios psicométricos. Estos estudios se basan en la constatación de que los miembros del público lego tienen poco que ver con probabilidades y resultados. Que los sujetos normalmente sobreestiman los riesgos asociados con sucesos infrecuentes, catastróficos e involuntarios y subestiman los riesgos asociados a eventos frecuentes, familiares y voluntarios. En general los sujetos perciben aquellas actividades involuntarias, desconocidas y con efectos retrasados como más arriesgadas que las opuestas (Fischhoff et al., 1978).

El tercero de los paradigmas es el sociocultural, que mantiene que la percepción del riesgo y nuestra actitud hacia él depende de los valores que mantenemos y de la cultura a la que nos adherimos, y que miembros de diferentes culturas atienden a diferentes categorías de riesgo (Douglas y Widaltsky, 1982). La teoría identifica cuatro culturas distintas denominadas; jerárquica, individualista, igualitarista y fatalista (Douglas y Galvez, 1990). Las fundamentales en este estudio son la jerárquica y la igualitaria. La estructura jerárquica, cuyo arquetipo es la burocracia, se caracteriza por una frontera marcada entre grupos sociales, y por una relación jerarquizada en el seno del grupo y una diferenciación de los estatus y de los roles. Constituye habitualmente el núcleo duro de cualquier sociedad. El sectarismo igualitario alude a pequeños grupos cerrados, aislados del resto de la sociedad, que instauran entre sus miembros unas relaciones igualitarias. Puede tratarse de sectas religiosas, de movimientos ecologistas o de organizaciones sindicales (Urteaga, 2012, 40-41).

Lo más importante de estos grupos es que no solo tienen distintas percepciones del riesgo, sino diferentes concepciones del conocimiento, de la naturaleza y del cuerpo humano, lo que guarda una profunda relación con las cosmovisiones de los grupos antivacunas analizadas más arriba. Por ejemplo, en la estructura jerárquica las fuentes de conocimiento son aquellas validadas a través de la autoridad, es decir, el saber científico institucional, mientras en la estructura igualitaria se desconfía de este tipo de saber y se tiende a buscar fuentes alternativas de conocimiento. Además, el saber puede ser adquirido de forma rápida a través de la lectura de las fuentes adecuadas sin necesitar un largo entrenamiento en la materia. La desconfianza que mantienen del saber oficial les lleva a mantener una cierta teoría conspiratoria.

Cada grupo mantiene una concepción de la naturaleza distinta: caprichosa, frágil, robusta o semirobusta. Para los igualitaristas la naturaleza es frágil. 
Los daños infligidos por los seres humanos son irreversibles, pero al mismo tiempo la naturaleza es bondadosa.

Como se señalaba más arriba los miembros de las diferentes culturas atienden a diferentes tipos de riesgo.

El tipo jerárquico teme todo lo que es susceptible de perturbar el orden social, especialmente las crisis política o económica, la guerra y la criminalidad. [...] En cuanto al enclave igualitario, se distingue por una aversión marcada hacia unos riesgos catastróficos e irreversibles, que amenazan, incluso a largo plazo, la supervivencia de la especie humana. (Urteaga, 2012, 48)

En cuanto al conjunto de valores sostenidos:

[e]1 enclave igualitario reúne unos individuos motivados, que comparten un sentimiento identitario fuerte. Implica la adhesión voluntaria de sus miembros. Para mantener la cohesión y la motivación del grupo, la secta debe mantener un tamaño reducido y asegurarse de que los miembros siguen siendo iguales entre sí, pero sobre todo cultivar su diferencia. Para ello, necesita enemigos que pueda criticar y combatir para llevar a cabo su cruzada: acusando el resto de la sociedad (especialmente los tipos jerárquico e individualista) de conducir el mundo a su pérdida, es decir focalizándose en los riesgos fundamentales, el tipo sectario denuncia todo el sistema social que le es exterior. (Urteaga, 2012, 48)

El grupo igualitario parece adecuarse con el grupo antivacunas que se ha venido analizando. En ese sentido, los mensajes que las autoridades enuncian acerca del riesgo son interpretados en forma totalmente contraria, dado que los valores que subyacen a uno y otro grupo son absolutamente diferentes. El enemigo de estos grupos, lo que les sirve de contraste en su definición, son los grupos de expertos asociados a la clase médica. El estado y la industria farmacéutica conspiran para beneficiar sus intereses económicos y vender vacunas con un objetivo exclusivamente económico. La naturaleza es bondadosa y nos proporciona los medios naturales para luchar contra la enfermedad. En ese proceso, el cuerpo humano se fortalecerá, mientras que la intervención química sobre él solo producirá debilidad. No confían además en el conocimiento médico institucionalizado ni en lo que se denominan hechos científicos. 


\section{Discusión}

Ernan McMullin (1987) habla de tres formas distintas en la clausura de una controversia: la que se produce en base a factores epistémicos, la que se produce en función de factores externos y el abandono de la misma. Pero lo cierto es que la controversia de las vacunas lleva abierta desde el siglo XIX y el rechazo a la vacunación obligatoria contra la viruela en Reino Unido (Williamson, 2007) y no parece que vaya a cerrarse. Tampoco es una controversia especialmente activa, los focos recaen sobre ella cuando algún nuevo suceso relacionado con las vacunas atrae la atención de los medios de comunicación, pero después cae en un estado latente. Aun así, las cifras de niños vacunados van cayendo lenta pero inexorablemente en todo el mundo occidental. De acuerdo a Eurostat, en el año 2014, Rumania tuvo una tasa de vacunación para el sarampión de un 89\%, Italia de un 80 y Francia de un 90. Para la rubeola Italia tuvo un $86 \%$ y Francia un $90 \%{ }^{23}$. Hemos de tener en cuenta que, por ejemplo, la tasa de inmunidad de grupo para el sarampión se sitúa en el 94\% (Vaqué, 2003), por lo que los tres países mencionados la habrían perdido, ello coincide con un repunte de los casos reportados. Un comunicado oficial de la Asociación Española de Pediatría recomienda el adelanto de la vacunación para los menores de un año que vayan a viajar a Francia, Grecia, Reino Unido, Gran Bretaña y Rumanía ante el rebrote de casos de sarampión en estos países ${ }^{24}$.

El factor determinante es la interpretación que cada uno de los grupos hace de los factores que intervienen en la controversia. En primer lugar, hay diferentes interpretaciones de la evidencia: qué es la evidencia de una reacción adversa, qué es la evidencia de la gravedad de las enfermedades prevenibles, qué es la evidencia de la eficacia de la vacuna. Según la OMS, para que pueda haber una atribución causal entre una vacuna y un efecto adverso de la misma deben cumplirse los siguientes criterios: (a) Relación temporal: la exposición a la vacuna ha de preceder al evento; (b) prueba definitiva de que la vacuna causó el evento (prueba clínica o de laboratorio); (c) causalidad basada en la evidencia poblacional (lo que a su vez implica que en los estudios epidemiológicos debe darse relación temporal, significación estadística, relación dosis respuesta, consistencia de la evidencia, especificidad y plausibilidad biológica); (d) plausibilidad biológica (la asociación debe ser compatible con

\footnotetext{
${ }^{23} \mathrm{https}: / /$ ec.europa.eu/health/vaccination/indicators_en

24 "Francia (casos de enero a mayo 2018: 2380), Grecia (2075), Italia (1715), Reino Unido (630) y Rumanía (504). En los últimos 12 meses (junio 2017 a mayo 2018), según el ECDC las mayores tasas, por orden decreciente corresponden a: Grecia $(282,2 / 100.000)$, Italia $(61,0)$, Rumanía $(61,0)$ y Francia $(38,6)$ ". http:// xurl.es/5iwds Consultado el 26/07/2018
} 
el conocimiento existente acerca de cómo funciona la vacuna); (e) consideración de explicaciones alternativas (enfermedades preexistentes, enfermedades nuevas, emergencia de una enfermedad determinada genéticamente, exposiciones a drogas o tóxicos, etc.); (f) evidencia previa de que la vacuna podría causar un evento similar (WHO, 2013, 6). Frente a esto las evidencias aportadas por los padres se suelen limitar a la ventana temporal sin que se cumplan el resto de los criterios. La negativa, por parte de las autoridades a calificar el evento como una reacción adversa debida a la inmunización es considerada un signo de conspiración. Esta es la fase IV de la investigación de un medicamento, que se corresponde con la farmacovigilancia, en la fase tres, los estudios se realizan con grupos de control, son los ensayos clínicos. Aquí la cuestión es la desconfianza en la evidencia debido a su fuente. En un ambiente de desconfianza hacia los expertos y la industria farmacéutica la evidencia que se propone en los mensajes provacunales está, según los grupos antivacunas completamente contaminada por los intereses económicos de las compañías farmacéuticas que solo buscan el beneficio económico. En esta fase se producen también los desacuerdos acerca de la metodología de la investigación: la evidencia estadística frente a la evidencia anecdótica.

En segundo lugar, están las diferentes interpretaciones de la ley. La legislación española coloca en un precario equilibrio el derecho a la libertad de elección de tratamiento y el derecho a un ambiente sano. Se enfrenta lo individual y lo público, los bienes privados frente a los bienes colectivos.

En tercer lugar, hay diferentes interpretaciones del riesgo. El mensaje de las autoridades públicas se centra en el cálculo riesgo beneficio, pero esta no parece ser la lógica que opera detrás de la percepción que tienen los padres del riesgo que corren sus hijos. Muchos pasaron el sarampión, la varicela o la paroditis de niños y las perciben como enfermedades inocuas. En lo que refiere a la concepción de la ciencia y la medicina es como las muñecas matrioskas. La controversia sobre las vacunas alberga en su seno una controversia acerca de la ciencia y esta una acerca de la medicina. Lo cierto es que si hay un punto en común en los movimientos antivacunas es la presencia en sus páginas web de informaciones acerca de tratamientos alternativos, especialmente para las reacciones adversas, basados en la homeopatía ${ }^{25}$. Las diferencias respecto a qué es la ciencia y quién es un científico conduce al debate de la experticia. Lo que está en juego son los límites del conocimiento paterno basado, tanto

\footnotetext{
${ }^{25}$ En un estudio en el que se analizaba el diseño y contenido de las páginas web antivacunas se halló que el $73 \%$ de las webs presentaban como argumento que las enfermedades han disminuido gracias a mejoras en la nutrición y en la higiene (no gracias a las vacunas) y que la homeopatía era un medio de reducir la severidad de las reacciones adversas a las vacunas (Wolfe et al., 2002).
} 
Claves para comprender la resistencia de los colectivos antivacunas: / Obdulia Torres González / 31 una controversia científico-tecnológica pública

en la observación cuidadosa de su hijo como en la adquisición de toda la información públicamente disponible. En el fondo se trata de la capacidad de participar en la controversia en calidad de expertos y en pie de igualdad con la clase médica y los investigadores o, al menos, que su conocimiento sea considerado complementario del de los expertos. Por ejemplo, los argumentos de los padres que correlacionan el autismo con la vacuna triple vírica se basan en esa observación privilegiada que tienen de su hijo. La cuestión es que esa experticia no es reconocida por los investigadores médicos. M. Fitzpatrick, un médico de atención primaria y padre de un niño autista, argumenta en su libro precisamente respecto a los límites del conocimiento paterno.

La experiencia de tener un niño con autismo te califica para hablar con autoridad sobre tu experiencia como padre de un niño autista pero no te da ninguna visión particular de la ciencia del autismo [...] El conocimiento científico moderno de cualquier disciplina es complejo y altamente especializado. La comprensión profesional de los investigadores y médicos es el producto de un largo proceso de estudio, práctica y experiencia. Tal conocimiento y experticia no puede ser adquirida a través de la lectura de artículos, bajando información de Internet o atendiendo conferencias ocasionales. (Fitzpatrick, 2004, 80)

En cuarto lugar y tal vez lo más llamativo de estas diferencias en interpretación sean las diferentes concepciones del riesgo. Bajo el paradigma psicométrico el riesgo aceptable de las vacunas está por debajo de actividades como esquiar o montar en bici, parece claro que más allá de las diferencias entre expertos y legos de los que da cuenta este paradigma, subyace otra diferencia entre individuos que queda inexplicada. En este caso el paradigma cultural nos proporciona una explicación plausible para los grupos antivacunas, y nuevamente, el factor determinante es esa cosmovisión del mundo en el que las relaciones grupales, de los individuos con la naturaleza y la desconfianza de las instituciones juegan un papel fundamental.

Las CCTP ponen en jaque la ciencia. Las investigaciones que se realizan para la toma y justificación de las decisiones son interpretadas de distintas maneras y los desacuerdos entre expertos alimentan la desconfianza del público hacia la ciencia. Una forma de explicar las divergencias se basa en las razones estructurales en que se construye la experticia en las CCTP (Aibar, $2002,114)$ Son las características propias de la ciencia regulativa la que explica las diferencias de opinión entre los expertos en ambos lados del campo de batalla. ¿Es la controversia de las vacunas un caso de ciencia regulativa? Para Dorothy Nelkin las controversias giran en torno a la cuestión del control 
político ¿quién controla las decisiones acerca de los desarrollos y aplicaciones de la ciencia? Si atendemos a las controversias analizadas por la propia Nelkim parece ser que sí, la mayor parte de las controversias científico tecnológicas públicas tienen en su base la toma de decisiones políticas sobre algún aspecto de la tecnología relacionado con la controversia: el uso de embriones para la investigación debe ser regulado, los defensores de los derechos animales piden poner límites a la investigación, los defensores y detractores de la gestación subrogada piden una norma que legalice o ilegalice la práctica, el agujero de la capa de ozono generó una actuación legislativa internacional bajo la forma del protocolo de Montreal, la apuesta por la energía nuclear se alimenta de las políticas públicas que apoyan o rechazan las energías renovables, etc. En el caso de las vacunas subyace en última instancia la decisión regulatoria: Australia acaba de sacar una normativa en la que se sanciona a los progenitores de aquellos niños que no han sido vacunados con 18 euros por quincena. Italia ha hecho obligatorias diez vacunas ${ }^{26}$, los niños nacidos a partir de 2017 deberán acreditar la vacunación para acceder a los centros escolares y el incumplimiento conllevará sanciones de hasta 7100 euros. En Francia los niños que no estén vacunados no podrán acceder al sistema educativo. En España el lamentable caso del niño de Olot puso sobre la mesa la obligatoriedad de las vacunas. Pero la controversia de las vacunas no cumple muchas de los rasgos de la ciencia regulativa propuestos por Jasanoff. De entre todas las características propuestas las más importantes son la incertidumbre y la inmediatez o limitación temporal. ¿Cuánta incertidumbre rodea al conocimiento de las vacunas? ¿Hay un auténtico disenso en la comunidad científica reconocida respecto a la seguridad y eficacia de las vacunas? ¿Existe una auténtica infradeterminación teórica? ¿Existe insuficiencia de datos para que el gobierno pudiera tomar una decisión regulatoria si este fuera el caso? La respuesta a todas estas cuestiones es negativa. No es la ciencia regulativa la que explica los desacuerdos entre los expertos, ni tan siquiera podemos situar a los dos grupos de expertos en pie de igualdad, son muy pocas las voces disidentes entre los expertos en epidemiología, salud pública o pediatría. La incertidumbre no se refiere tanto al conocimiento como al conjunto de criterios aceptados por la comunidad investigadora y las agencias de salud pública para atribuir causalidad. Lo que explica los desacuerdos son las diferencias en interpretación socialmente determinadas por una cosmovisión diferente. Esto

\footnotetext{
${ }^{26}$ Las vacunas son: poliomielitis, difteria, tétanos, tosferina, hepatitis B, Haemophilus influenzae tipo b, sarampión, rubeola, parotiditis y varicela. Circolare recante prime indicazioni operative per l'attuazione del decreto-legge 7 giugno 2017, n.73, recante "Disposizioni urgenti in materia di prevenzione vaccinale" (Gazzetta ufficialen.130 del 7-6-2017).
} 
a su vez nos permite entender las diferencias de este sector concreto dentro del espectro de las actitudes ante la vacunación.

\section{Conclusión}

Tras la muerte del niño de Olot, las intervenciones en los medios de comunicación de las autoridades sanitarias hacen surgir la pregunta, qué "información veraz" pretendía la sanidad pública distribuir entre los padres, que tuviera más peso que la muerte de un niño por una enfermedad fácilmente prevenible con una vacuna. Pero lo cierto es que a pesar de un suceso de esa magnitud 47 niños del entorno continuaban sin ser vacunados. Esto va en contra de desarrollos recientes en ciencia cognitiva que postulan que las creencias provacunación son contraintuitivas (y por ello más difíciles de difundir) y afirma que uno de los mecanismos a través de los cuales las creencias provacunación pueden llegar a ser más intuitivas radica en la preponderancia de la enfermedad: "ver a las personas no vacunadas ponerse enfermas mientras las vacunadas permanecen sanas ... los mensajes que juegan con la prominencia de la amenaza de la enfermedad se encuentran entre los únicos que han mostrado alguna promesa de reducir la duda ante la vacunación" (Milton y Mercier, 2015, 634).

Los factores que las autoridades públicas manejan tienen que ver con lo que tradicionalmente se ha llamado el modelo del déficit, el público en general no tiene los conocimientos necesarios para entender el conocimiento científico ${ }^{27}$ que está detrás de los programas de inmunización, y tampoco parecen ser capaces de entender el riesgo que corren sus hijos al no ser vacunados. Frente a esto, a lo largo de la investigación surge como elemento determinante en la conformación de la controversia el conjunto de valores mantenido por la comunidad antivacunas. Son los valores y creencias del grupo lo que determina su actitud hacia el riesgo, su consideración de lo que es la información relevante, la ciencia, la medicina y el cuidado adecuado del cuerpo. Por su parte el conjunto de expertos que apoya a los movimientos antivacunas, invoca un paradigma farmacológico y médico distinto para legitimar su discurso e intentar entrar en la comunidad científica como expertos.

El mejor abordaje de las vacunas es a través de las controversias científico tecnológicas dado que bajo este enfoque, y analizando el discurso de los

27 "Hay evidencia en la literatura reciente que sugiere que las élites más educadas en Netherlands, Estados Unidos y Canadá sostienen visiones escépticas respecto a la vacunación” (Larson, 2016, 300). 
contendientes emergen los factores, sociales, culturales y morales que determinan la evidencia científica admisible.

El problema de las vacunas es un problema social en el que todos estamos implicados. La inmunidad de grupo que sirve de escudo a aquellas personas que no pueden ser vacunadas corre peligro en muchas regiones europeas, aunque todavía las tasas vacunales en España son altas. La comunidad científica representada por el estamento médico se halla atrincherada en una posición defensiva, que les hace negar o poner en duda sistemáticamente todo aquello que tenga que ver con un efecto adverso relacionado con una vacuna, o la posible seguridad y eficacia de las mismas, lo cual también va en detrimento de la seguridad de la población. En este sentido habría que mantener un sano escepticismo.

Es altamente improbable que la controversia de las vacunas se cierre alguna vez, dado que las autoridades sanitarias pretenden clausurarla en base a una evidencia que no cuenta como tal para el resto de los actores implicados. Es una controversia anclada en cosmovisiones, en ideas diferentes acerca de los que es una buena vida y de cuál debe ser la relación de los seres humanos con el entorno. Tal vez, desde dentro de esa cosmovisión pueda emerger un discurso distinto, que no entienda las vacunas como una agresión contra una elección personal y se prioricen los valores de la solidaridad social.

\section{Referencias}

Aibar, E. (2002). "El conocimiento científico en las controversias públicas". En E. Aibar y M. Quintanilla, Cultura tecnológica. Estudios de ciencia, tecnología y sociedad. Barcelona: Ed. Horsori, 105-126.

Carozzi, Julia (1993). "Definiciones de la New Age desde las Ciencias Sociales". Boletín de Lecturas Sociales y Económicas, Año 2, № 5, 19 24.

Charlton, B. (1996). "The Scope and Nature of Epidemiology". Journal of Clin Epidemiol, Vol. 49, No. 6, 623-626.

Douglas, M., Wildavsky, A. (1982). Risk and Cuilture. California: University of California Press.

Douglas, M., Galvez, M. (1990). "The self as risk taker: a cultural theory of contagion in relation to AIDS". Sociological Review, Volume 38, Issue 3, 445-464. 
Claves para comprender la resistencia de los colectivos antivacunas: / Obdulia Torres González / 35 una controversia científico-tecnológica pública

Fischhoff, B. et al. (1978). "How safe is safe enough? A Psychometric Study of Attitudes towards technological Risks and Benefits". Policy Sciences $9,127-152$.

Fitzpatrick, M. (2004). MMR and Autism. What Parents Need to Know. London: Routledge.

Gieryn, T. (1995). "Boundaries of science". En S. Jasanoff et al. Handbook of Science, Technology and Society. London: Sage Publications, 393443.

Hervieu, B., Léger, D. (1979). Le retour a la nature, «au fond de la forêt... l'Etat». Paris: Le Seuil.

Ibargüen, J. et al. (2004). "Neorrurales: dificultades durante el proceso de asentamiento en el medio rural aragonés", Informes 5 (2004-3). ISBN 978-84-92582-32-7.

Instituto de Salud Carlos III (s.f.). Estudio seroepidemiológico: situación de las enfermedades vacunables en España. Ed. Instituto de Salud Carlos III, Centro Nacional de Epidemiología.

Jasanoff, S. (1987). "Contested Boundaries in Policy-Relevant Science". Social Studies of Science, Vol. 17, No. 2 (May, 1987), 195-230.

Kahneman, D., Tversky, T. (1987). "Teoría Prospectiva; un análisis de la decisión bajo riesgo". Infancia y aprendizaje, 30, 95-124.

Larson, H.J. et al. (2016). "The State of Vaccine Confidence 2016: Global Insights Through a 67-Country Survey”. EBioMedicine, 12, 295-301.

Leach, M., Fairhead, J. (2007). Vaccine Anxieties Global Science, Child Health and Society. London: Eartscan.

Martínez-Diz, S., Martínez Romero, M., Fernández-Prada, M., Cruz Piqueras, M., Molina Ruano, R., Fernández Sierra, M.A. (2014). "Demandas y expectativas de padres y madres que rechazan la vacunación y perspectiva de los profesionales sanitarios sobre la negativa a vacunar". Anales de Pediatría, 80 (6), 370-37.

Martin, B., (2014). Controversy Manual. Sparsnäs, Sweden: Irene Publishing.

Martin, B., Richards, E. (1995). "Scientific Knowledge, Controversy, and Public Decision Making". En S. Jasanoff et al. (eds.), Handbook of Science and Technology Studies. California: Sage Publications. 
Mazur, A. (1981). The Dynamics of Technical Controversies. Whasington DC: Communication Press.

McKee, Ch., Bohannon, K. (2016). "Exploring the Reasons Behind Parental Refusal of Vaccines". BSJ Pediatr Pharmacol Ther. Mar-Apr; 21(2): 104-109. DOI: 10.5863/1551-6776-21.2.104.

McMullin, E. (1987). "Scientific Controversies and its termination". En H. Engelhardt y A. Caplan, Scientific Controversies. Case studies in the resolution and closure or disputes in science and technology. Cambridge: Cambridge University Press.

Meszaros, Jacqueline R., Asch, David A., Baron, Jonathan, Hershey, John C., Kunreuther, Howard, Schwartz-Buzaglo, Joanne (1996). "Cognitive Processes and the Decisions of Some Parents to Forego Pertussis Vaccination for Their Children". J Clin Epidemiol, Vol. 49, No. 6, 697-703.

Miller, E. (2015). "Controversies and challenges of vaccination: an interview with Elizabeth Miller". BMC Medicine, 13: 267.

Milton, H., Mercier, H. (2015). "Cognitive Obstacles to Pro-Vaccination Beliefs". Trends in Cognitive Sciences, Vol. 19, No. 11, 633-636.

Ministerio de Sanidad, Política Social e Igualdad (2011). Análisis de situación de terapias naturales. Documento de trabajo.

Mora, Joan (2008). "Homeopatía e inmunología". Boletín informativo de la Liga para la Liberta de Vacunación, $\mathrm{n}^{\circ} 16$.

Morgan, M. (2010), "Voice and the Facts and Observations of Experience". En W. González, New Methodological Perspectives on Observation and Experimentation in Science: Coruña: Netbiblo.

Nelkin, D. (1992). Controversy. Politics of technical decisions. California: Sage Publications.

Nogué i Font, J. (1988). "El fenómeno neorrural”. Agricultura y Sociedad, $n^{\circ} 47,145-175$.

Rogers, A., Pilgrim, D. (1995). "The risk of resistance: Perspectives on the mass Childhood Immunisation Programme". En J. Gabe (ed.), Medicine, Health and Risk: Sociological Approaches: Oxford: Blackwell.

Urteaga, E. (2012). "Los determinantes culturales en la percepción social del riesgo". Argumentos de Razón Técnica, $\mathrm{n}^{\circ}$ 15, 39-53. 
Vaqué, J. (2003). "Inmunidad de grupo significado e importancia". Revista Clínica Electrónica en Atención Primaria. URL: https://ddd.uab.cat/ pub/rceap/rceap_a2005m11n8/rceap_a2005m11n8a2.pdf. Acceso 13/07/2018.

Weber, E. U. (2011). "Risk: Empirical Studies on Decision and Choice". En N. J. Smelser, P. B. Baltes, International Encyclopedia of the Social \& Behavioral Sciences. Pergamon: Elsevier.

Weinberg, A. (1974). "Science and transcience". Minerva 10(2): 209-222.

WHO (2013). Causality assessment of adverse event following immunization (AEFI): user manual for the revised WHO classification. WHO Library Cataloguing-in-Publication Data.

Williamson, S. (2007). The Vaccination Controversy: The rise, reign and fall of compulsory vaccination for smallpox. Liverpool: Liverpool University Press.

Wolfe, R.M., Sharp L.K., Lipsky, M.S. (2002). "Content and Design Attributes of Antivaccination Web Sites". Journal of American Medical Association, Vol. 287, $\mathrm{n}^{\circ}$ 24, 3245-3248. 
